All the babies found to have ROP were regularly followed up in both our high risk clinic and in Ophthalmology outpatient department for one year.

Results Incidence of ROP was $23 \%$ in our study. Incidence was $38 \%$ in the 1 st group $\& 8 \%$ in the 2 nd group.

Most common findings were stage III ROP (39\%) and zone II ROP (70\%). 3 (13\%) had APROP (Aggressive posterior ROP) and 2 (8.7\%) had retinal detachment.

The incidence of ROP increased as the birth weight and period of gestation decreased- no ROP was found in gestation $\geq 35$ weeks and birth weight $>1.828 \mathrm{~kg}$.

In our study, oxygen administration through mechanical ventilator or CPAP, sepsis, therapy with surfactant, apnea, PRBC transfusion, NEC and birth asphyxia were found to be significant risk factors. The risk of ROP is more in RDS, IVH and only head box oxygenation but the risk is not significant. We have not found any ROP in the babies who have undergone exchange transfusion.

$13(57 \%)$ babies had spontaneous regression of ROP and rest $10(43 \%)$ required some intervention. To summarize the intervention $(\mathrm{n}=10), 5(50 \%)$ responded to LASER only, 3 $(30 \%)$ required intravitreal injection with Bevacizumab (antiVEGF) following LASER, 1 (10\%) required TPPV (Trans Pars Plana Vitrectomy) in left eye for ROP stage IVb along with LASER and 1 (10\%) baby required all the three- LASER, intravitreal Bevacizumab and left sided TPPV.

Conclusions Thus the need for a routine screening program for the detection of ROP in preterm and sick neonates with risk factors is very essential in our clinical settings. Prevention of blindness from ROP can be very effective through early detection and urgent treatment. This needs awareness among neonatologists and pediatricians for referral to the ophthalmologists at appropriate age of the baby.

\section{CAN WE REDUCE NEONATAL ADMISSIONS DUE TO JAUNDICE?}

Sonal Datir, Gopa Sarkar. UK

\subsection{6/bmjpo-2021-RCPCH.227}

Background Neonatal hyperbilirubinemia is a common cause for neonatal admission in term and preterm infants. These infants are primarily managed on postnatal wards; however, they require admission to neonatal unit due to jaundice above the exchange level, rapidly increasing bilirubin levels, pathological jaundice or sepsis.

Failure to initiate and establish adequate breastfeeding can play an important role in development of severe jaundice. Face-to-face professional support has been shown to increase breastfeeding success.

Objectives The objectives of this project included evaluation of the management of infants admitted to neonatal unit with jaundice including the feeding practices; and identifying the areas of improvement to reduce admissions.

Methods This was a retrospective observational project including infants born at $\geq 35$ weeks admitted to neonatal unit at District Hospital with a diagnosis of jaundice from January 1, 2017 to December 2018. Data was collected using proforma, medical records and blood results on computer system.

Results A total of 519 infants $\geq 35$ weeks were admitted to the neonatal unit of which $12 \%(60)$ infants were admitted due to jaundice. $42 \%(25)$ were preterm and $58 \%(35)$ were term infants.

The risk factors for jaundice were identified as male infant (66\%), first born (49\%), gestation, prematurity, and breast fed babies.

The mean birth weight was 2892.5 grams and 20\%(12) were low birth weight. 35\%(21) infants were admitted from home (average 3.9 days) and 65\%(39) from postnatal wards.

$27 \%(16)$ infants had jaundice $<24$ hours. 6 infants had $>$ $10 \%$ weight loss on admission. DAT was positive in $23 \%(14)$ infants of which 8 infants presented $<24$ hours.

Sepsis was suspected in $72 \%$ (43) infants but was proven in none.

Only 20\%(12) received lactation support on the postnatal ward prior to admission whereas $43 \%(26)$ did not receive any support. 36 infants were exclusively breastfed, 13 were formula fed and 11 were mixed-fed. After admission, formula feeds were added to 32 infants while 4 infants exclusively breast fed. 10 infants were given intravenous fluids.

The causes of jaundice included prematurity(25), ABO incompatibility(11), Rh incompatibility(3), poor feeding or exaggerated jaundice(25).

33 infants had bilirubin above exchange line, and required a mean of 24 hours (range 6- 144 hours) of phototherapy. None of them required immunoglobulins or exchange transfusion.

The mean length of stay was 3 days (range 1-14 days) and there was a remarkable decrease in breast feeding as only 7 (11.6\%) infants were breast fed on discharge.

Conclusions There is a scope to decrease the admissions due to jaundice by optimising the postnatal support on the postnatal wards and community in the presence of risk factors.

Transitional care for late preterm infants is important to reduce admission to the neonatal unit thereby reducing the separation of mother and baby.

The opportunities to support breast feeding on postnatal ward and neonatal unit are often missed.

Effective measures should be taken to promote lactation support at all levels.

\section{SYRINGE AIR FLUSH TECHNIQUE ELIMINATES SURFACTANT REFLUX AS A LIMITING FACTOR IN USING HIGHER VOLUME SURFACTANT FOR MINIMALLY INVASIVE SURFACTANT THERAPY}

Karthikeyan Gengaimuthu. United Arab Emirates

\subsection{6/bmjpo-2021-RCPCH.228}

Background Minimally Invasive Surfactant Therapy or Less Invasive Surfactant Administration (LISA) is the preferred way of administration of surfactant in neonates. Surfactant reflux resulting in ineffective surfactant delivery is considered a limiting factor in administering a higher volume surfactant like bovine surfactant by MIST.

Objectives To analyze if syringe air flush technique as incorporated in our MIST procedure protocol eliminates surfactant reflux with higher dose volume bovine surfactant in our cohort of babies that received surfactant by MIST.

Methods Syringe air flush after the surfactant administration is incorporated in our MIST procedure protocol, the default standard of administering surfactant in our neonatal units. Both porcine surfactant $(1.5 \mathrm{ml} / \mathrm{kg})$ and bovine surfactant 
(survanta $4-8 \mathrm{ml} / \mathrm{kg}$ ) are used in our babies. We performed a subgroup analysis comparing the MIST success rate and the relevant clinical outcomes in our prospective observational cohort of MIST babies (14 times in 13 babies) that received porcine surfactant $(n=9$, one time each in nine babies) versus those that received bovine surfactant $(n=5$, one time each in three babies and two times in 1 baby).

Results Nine babies (gestation 27 - 36 weeks and birth weight $0.95 \mathrm{~kg}$ to $2.4 \mathrm{~kg}$ ) received Curosurf $(1.5 \mathrm{ml} / \mathrm{kg}, 200 \mathrm{mg} / \mathrm{kg}$ ) by MIST one time in each baby. The median age of administration of Curosurf was $12.5 \mathrm{~h}$. Conduits used for MIST were by infant feeding tube in eight babies and LISA (Less Invasive Surfactant Administration) catheter in one baby. Four babies (gestation 27 - 34 weeks and birth weight $1.04 \mathrm{~kg}$ to 2.81 $\mathrm{kg}$ ) received Survanta $(4-8 \mathrm{ml} / \mathrm{kg}, 100-200 \mathrm{mg} / \mathrm{kg})$ five times by MIST one time each in three babies and two times in one baby. The median age of administration of survanta was $13 \mathrm{~h}$. The conduits used for the MIST were infant feeding tube $\mathrm{s}$ on two occasions, LISA catheter once and $2.0 \mathrm{~mm}$ Endotracheal Tube with surfactant filled syringe directly attached to the syringe hub on two occasions in one baby. Syringe air flush and checking for surfactant reflux was done as per MIST procedure protocol on all occasions after the instillation of surfactant. Success rate of MIST procedure was $100 \%$ irrespective of surfactant preparation. Equally beneficial clinical outcomes are seen in babies receiving bovine surfactant MIST and porcine surfactant MIST.

Conclusions Surfactant reflux is nullified with the incorporation of syringe air flush technique in MIST thereby ensuring equally better clinical outcomes with higher volume bovine surfactant versus porcine surfactant in this cohort. A higher dose volume surfactant can be effectively delivered by MIST.

\section{PATIENT PERSPECTIVES REGARDING ONLINE CONSULTATIONS DURING THE COVID-19 PANDEMIC}

John Cheri Mathews, Deepa Elizabeth Mathew, Shipra Mathur, Natasha Susan John, Suneela Nayak, Vinay Bothra. India

\subsection{6/bmjpo-2021-RCPCH.229}

Background Introduction: COVID-19 and the associated lockdown has prevented patients from accessing healthcare professionals through the traditional face-to-face clinic visits. Modes of consultation have therefore adapted to overcome this barrier; virtual or online consultations have become a popular alternative for people with access to technology. The literature on patient perspectives about this mechanism is still evolving.

Objectives Aim: To explore patient perceptions regarding virtual consultations

Methods A voluntary online survey instrument using a mix of quantitative and qualitative questions was administered to patients across 3 major cities using a social media platform, WhatsApp. The aim was to explore the characteristics of users, perceived advantages and disadvantages of online consultations and patient satisfaction.

Results There were 461 respondents (M 51.4\%: F 48.6\%) that had consulted doctors online. $91 \%$ of them lived in 8 major metro cities. Interestingly, over $80 \%$ respondents had never sought online consultation before the COVID-19 pandemic. $52 \%$ patients accessed multiple (2-3) consultations in the 10 months since the start of the lock-down in March 2020. While $62 \%$ consulted their regular doctor, $19 \%$ accepted recommendations from friends and family and 10\% used online platforms. $45 \%$ of consultations were via videocalls, 28\% through WhatsApp and 20\% via telephone calls. Prescriptions were provided via WhatsApp in $41 \%$ cases, online portals in 32\%, email in $13 \%$ and a photograph of handwritten prescription in $13 \%$ cases. The vast majority (90\%) felt that the time provided by the doctor was adequate. $55 \%$ of patients paid via $G$ pay while $28 \%$ were prepaid through online portals. There were no audio or video connectivity issues in $90 \%$ cases. $13 \%$ patients had to go for a faceto-face consultation within 7 days of the online consultation as the clinical problem had not been resolved adequately. Patients felt that the main advantages of online consultations included a lower risk of infection (77\%), reduced waiting time $(57 \%)$ and travel time (58\%). The main disadvantages cited included a lack of physical examination (73\%), a perception that this was not as satisfying as a face-to-face consultation (36\%), inability to adequately communicate their problem (24\%) and an inability to show past reports (13\%). $78 \%$ patients rated their online consultations as either a $4 / 5$ or $5 / 5$ satisfaction level. Given the choice after the pandemic, almost two-thirds (64\%) felt they would still prefer face-face consultations.

Conclusions For the vast majority of patients (80\%), this pandemic provided the first ever opportunity to choose a virtual platform to seek clinical care. The high level of satisfaction for online consultations suggests that this mechanism of patient-provider clinical service provision might well be an increasingly popular intervention after restrictions are lifted. As digital penetration improves, weaknesses identified in the current system of online consultations (e.g., inability to conduct physical examinations and inadequate communication) could be mitigated through evolving technologies like digital stethoscopes and better communication tools. It brings into focus the need for regulations to keep pace with this rapidly evolving trend to ensure that the virtual patient-provider interaction remains a safe, secure and confidential way to access clinical services.

\section{RESPIRATORY VIRUS INFECTION-ASSOCIATED MORTALITY AMONG CRITICALLY ILL CHILDREN: A RETROSPECTIVE SINGLE-CENTRE COHORT STUDY}

Divyapoorani Ravichandran, Kian Boon, Joel Lim, Poh Hui Wee, Chee Fu Yung, Jan Hau Lee, Kee Thai Yeo. Singapore

\subsection{6/bmjpo-2021-RCPCH.230}

Background Limited data from Singapore indicates that respiratory virus infection (RVI) burden is high and comparable to other settings in temperate countries. Acute lower respiratory infections from RVIs are important contributors to mortality in children $<5$ years of age, with most deaths occurring in the first year of life.

Objectives This study aims to describe the burden and epidemiology of RVI-associated mortality among children admitted to the paediatric intensive care unit (PICU).

Methods We performed a linkage analysis of PICU mortality database with laboratory confirmed RVI database of patients $<18$ years old from 2010 to 2019. RVI-associated mortality was defined as cases with a laboratory confirmed RVI from any respiratory sample within 14 days prior to the certified date of death. 